\title{
COMPOSITION, DIVERSITY AND FORAGING GUILDS OF AVIFAUNA IN A SUBURBAN AREA OF SOUTHERN WEST BENGAL, INDIA
}

\author{
Shiladitya Mukhopadhyay and Subhendu Mazumdar
}

\begin{abstract}
Mukhopadhyay S., Mazumdar S. 2017. Composition, diversity and foraging guilds of avifauna in a suburban area of southern West Bengal, India. Ring 39: 103-120.

Avian communities are very good indicators of any ecosystem. Despite the alarming consequences of rapid urbanization, studies of avian diversity in the human-dominated landscapes of India are very few. Therefore, we studied the avian assemblage of Bongaon in southern West Bengal, India, a suburban area whose avifauna has thus far remained undocumented. Bird surveys were carried out from June 2015 to May 2016, following the fixed-radius $(25 \mathrm{~m})$ point count method together with opportunistic observations. We recorded 119 avian species belonging to 53 families. Ardeidae was the most diverse avian family in the study area ( $\mathrm{RDi}$ value $=5.882$ ). Among the recorded avifauna, 89 species were resident, 26 species were winter visitors, three species were summer visitors, and one species was a passage migrant. Species richness of the resident and passage migrant species did not vary seasonally, while the winter and summer visitors displayed significant seasonal variation. In this suburban area, the species richness of feeding guilds varied significantly. Most birds were insectivorous (41.2\%), followed by carnivorous $(24.4 \%)$, omnivorous $(18.5 \%)$, granivorous $(7.6 \%)$, frugivorous $(3.4 \%)$, nectarivorous $(3.4 \%)$ and herbivorous species (1.7\%). Maximum species richness was recorded in November and minimum species richness in July. Black-headed Ibis Threskiornis melanocephalus and Alexandrine Parakeet Psittacula eupatria are two near-threatened species found in this region. Interestingly, six species having a globally declining trend are still very common in the study area. Long-term studies are required to monitor any change in the avian communities of this suburban landscape resulting from urbanization.
\end{abstract}

S. Mukhopadhyay, Post Graduate Department of Zoology, Barasat Government College, Barasat, West Bengal, India; S. Mazumdar (corresponding author), Department of Zoology, Shibpur Dinobundhoo Institution (College), Howrah, West Bengal, India. E-mail: subhendumazumdar@gmail.com

Keywords: avifauna, bird community, diversity, feeding guild, suburban area 


\section{INTRODUCTION}

Birds are one of the most widely distributed animal taxa, living in diverse landscapes across continents. They show a substantial variety of distribution patterns and often prefer to live in heterogeneous environments. Birds generally colonize in an area with suitable resources for their survival (Veech et al. 2011), performing a variety of functional roles and providing vital ecological services in various ecosystems (Whelan et al. 2008, Sekercioglu 2006, 2012). Changes in vegetation composition can affect the quality and quantity of habitats for birds in terms of food, water and cover, which can further affect their diversity, abundance and distribution (Western and Grimsdell 1979). Therefore, the avian diversity of any ecosystem is considered to be an important indicator of its health (Lawson et al. 1998, Gregory et al. 2003, 2008). Bird surveys (biological monitoring) may be better than chemical or radiological monitoring of environmental health, as they are simple and relatively inexpensive and provide information on the possible impact of environmental change on the flora and fauna thriving at a given site, while the information derived from chemical testing merely reflects its contamination status (Khan et al. 2013). Thus the bird species assemblages characteristic of a given ecosystem can be a useful predictor of its integrity and functions.

Unfortunately, the global diversity of birds is continually decreasing, primarily due to anthropogenic disturbances (Rapoport 1993) and climate change (Chen et al. 2011, Sekercioglu et al. 2012). The IUCN Red List of endangered birds has already recognized 1,226 threatened species globally, of which 88 are found in India (BirdLife International 2010). Urbanization is a major threat to many avian species throughout the globe (Pickett et al. 2001, Hansen et al. 2005). Progressive urbanization often leads to biotic homogenization, whereby a few widespread and generalist species replace diverse avifauna (McKinney and Lockwood 2001, Crooks et al. 2004). Despite the alarming consequences of rapid urbanization on various biodiversity components worldwide, human-dominated areas are studied less than natural habitats (Marzluff et al. 2001). However, with greater understanding of the deleterious influence of urbanization on biodiversity and environmental processes (Miller and Hobbs 2002, McKinney 2002), in the last few decades urban and suburban habitats have gained importance in biodiversity conservation efforts in the United States (Blair and Johnson 2008). Nevertheless, despite this increasing trend in documentation of avian diversity in various cities and suburbs of western countries, similar studies in the human-dominated landscapes of India are almost non-existent. As a result, the avian diversity of many such areas still remains undocumented. It is crucial to assess the avian diversity of any ecosystem together with its role in maintaining various ecosystem functions (Lawson et al. 1998). The relative values of different habitats together with their conservation importance can be preliminarily assessed by investigating the diversity of the birds present in them (Bensizerara et al. 2013). Therefore, the present study was carried out to document the diversity of birds of a suburban area where no such work had previously been done. The aims of our study were (1) to document the composition and richness of avian species and (2) to assess the seasonal change in the species composition of birds in a suburban area of southern West Bengal, India. 


\section{METHODOLOGY}

\section{Study area}

This study was conducted in Bongaon and the adjoining areas (about $23.07^{\circ} \mathrm{N}$, $88.82^{\circ} \mathrm{E}$ ), in West Bengal, India (Fig. 1). This suburban area lies on the banks of the Ichhamati River near the India-Bangladesh border. The topography of the area comprises scattered horticultural gardens, orchards, bamboo bushes, shrubs, herbs and vast stretches of irrigated paddy fields interspersed with expanding human settlements. The average annual rainfall of the study area is about $1,579 \mathrm{~mm}$, temperature ranges from $41^{\circ} \mathrm{C}$ in summer to $10^{\circ} \mathrm{C}$ in winter, and relative humidity varies between $50 \%$ and $90 \%$. The climate of the study area comprises four seasons: summer (March-May), monsoon (June-August), post-monsoon (September-November) and winter (December-February).

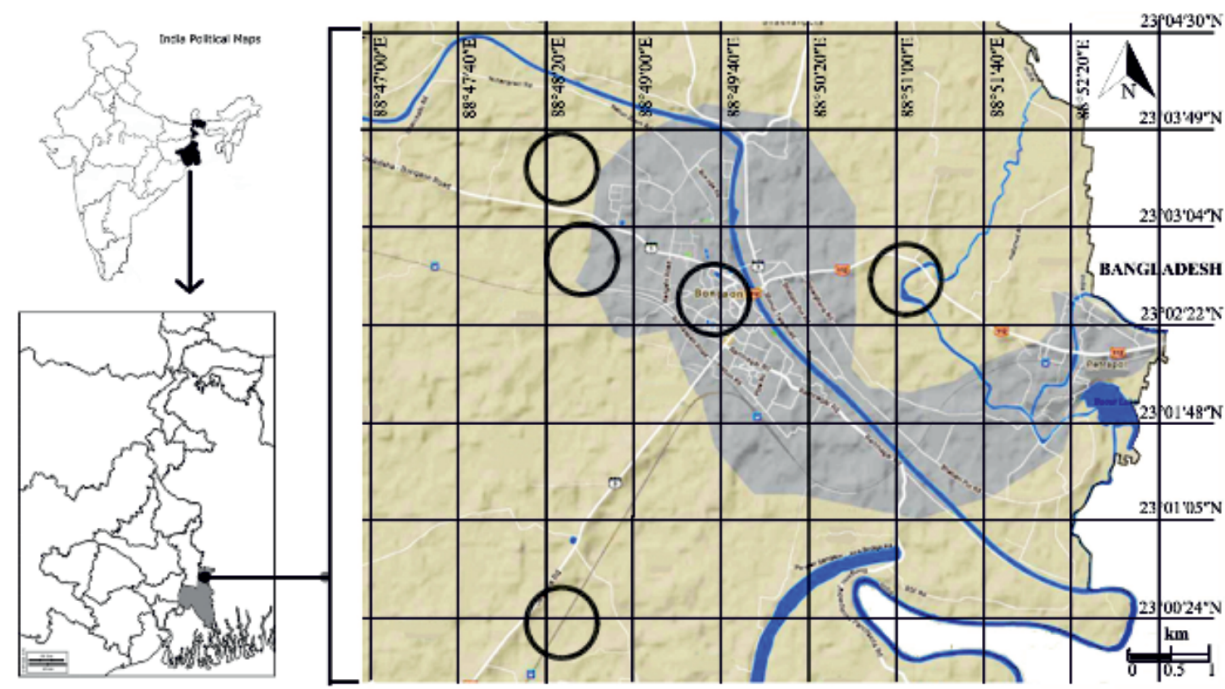

Fig. 1. Map of the study area Bongaon, West Bengal, India with the location of the study sites indicated as circles (Map source: Google)

\section{Methods}

Avifaunal surveys were carried out every week from June 2015 to May 2016. Field surveys were conducted in the morning (between 6:00 and 9:00) and in the evening (from 15:00 to 18:00/19:00, depending on the day length), when birds were found to be most active. During each visit, the fixed-radius point count method was used to record the species richness of avifauna at each count station (Bibby et al. 2000, Sutherland 2006). Observation points were randomly placed in different habitats. We recorded bird species (seen or heard) within a $25 \mathrm{~m}$ radius of each of these point count stations in a $360^{\circ}$ arc for 10 minutes. In addition, opportunistic observations of birds at other 
times and in other places were included in order to produce a comprehensive checklist of the avifauna of the study area. Field visits were carried out on foot only on days with suitable weather conditions (i.e. in the absence of rain or strong wind). Birds were observed either with the unaided eye or using binoculars (Olympus 7x21 PS III), and photographs were taken with a digital camera (Nikon P600) for documentation of the avifauna.

Birds were identified using guides by Ali and Ripley (1987) and Grimmett et al. (2011). The systematic position (order and family), common name and scientific name of each species were assigned following Praveen et al. (2016). Based on their seasonal dispersal pattern, birds were classified as resident (R), summer visitor (SV), winter visitor (WV) or passage migrant (P), following Grimmett et al. (2011). We also assigned a local status to each species following Khan and Naher (2009), where very common (Vc) bird species were recorded on $80-100 \%$ of field visits, common (Co) species on $50-79 \%$ of field visits, fairly common (Fc) on $20-49 \%$ of field visits and rare $(\mathrm{Ra})$ on less than $20 \%$ of the field visits. The conservation status of birds and their global population trend were taken from the IUCN Red List (Del Hoyo et al. 2014). Feeding guild is defined as a group of species with similar foraging habits (Hutto 1985). Our observed avian species into seven guilds, i.e. carnivore (Car), omnivore (Omn), frugivore (Frug), herbivore (Herb), nectarivore (Nect), granivore (Gran) and insectivore (Ins), following Ali and Ripley (1987).

\section{Data analysis}

Species richness data was pooled together within four seasons - summer (March-May), monsoon (June-August), post-monsoon (September-November) and winter (December-February), to test the seasonal pattern of the avian assemblage in the study site. The relative diversity (RDi) of families was calculated using the following formula (Torre-Cuadros et al. 2007):

$$
R D i=\frac{\text { Number of bird species in the family }}{\text { Total number of species }} \times 100
$$

Non-parametric Kruskal-Wallis tests with subsequent post-hoc multiple pair-wise comparisons were carried out separately to test the seasonal differences between species richness across different foraging guilds, as well as between residents, winter visitors, summer visitors and passage migrants. Statistical tests were computed using XLSTAT software (Addinsoft Corporation, Paris, France).

\section{Results}

A total of 119 species of birds belonging to 16 orders and 53 families were recorded during the study period (Table 1). The greatest number of avifaunal species belonged to the order Passeriformes (52 species), followed by the order Pelecaniformes (11 species), Charadriiformes (10 species), and others. More than half $(61.3 \%)$ of the species found during the study belonged to one of three orders (Passeriformes, Pelecaniformes and Charadriiformes). Analysis of relative diversity revealed that Ardeidae was the most diverse avian family in the study area ( 7 species, $R D i=5.88)$, followed 


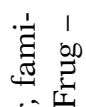
年

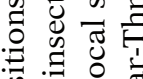

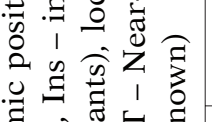

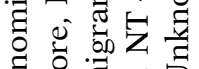

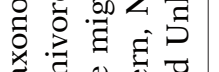

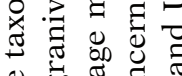

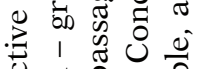

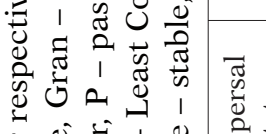

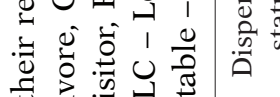

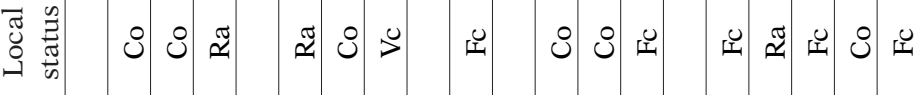

$=$

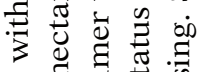

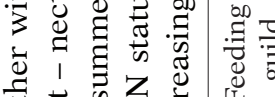

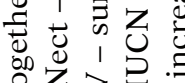

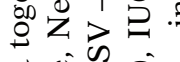

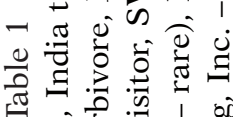

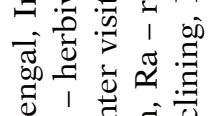

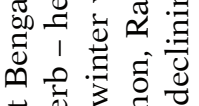

可

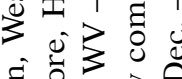

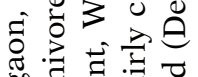

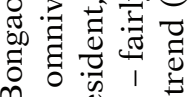

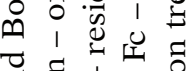

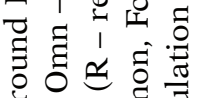

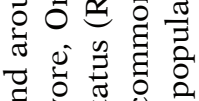

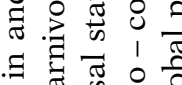

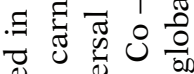

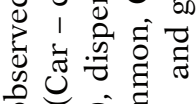

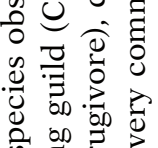

总

y @ @

y

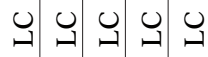

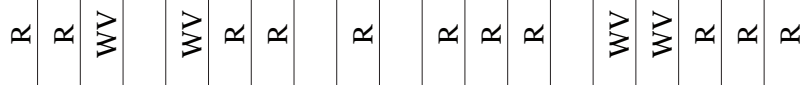

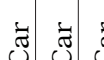

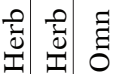

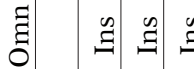

氞题氞言言

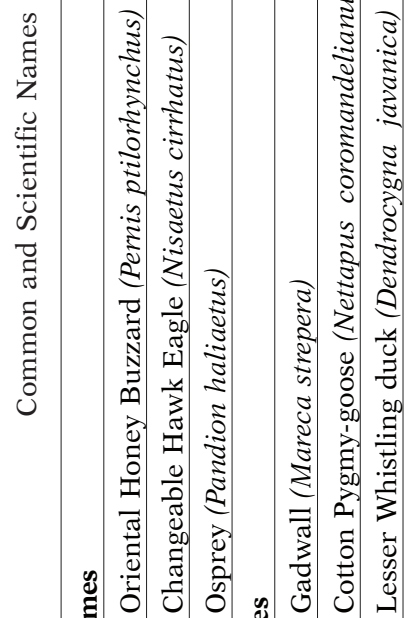

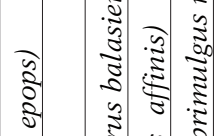

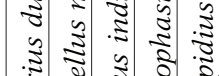

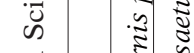

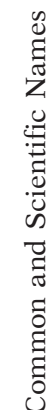

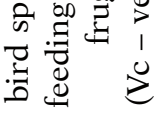

量宝

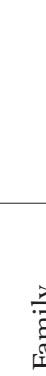

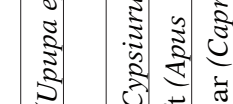

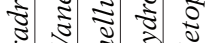

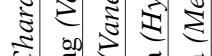

ट)

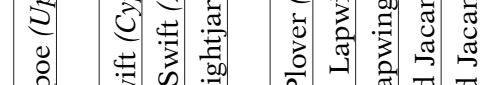

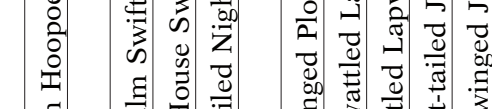

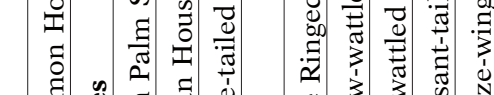

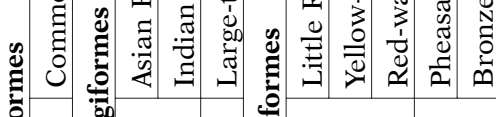
ถูป

.

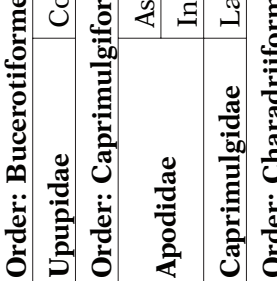

胥

త্] 


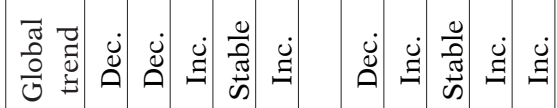

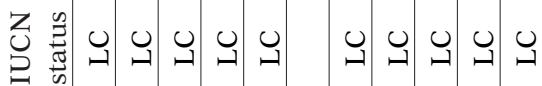

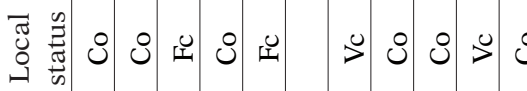

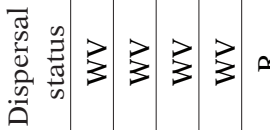

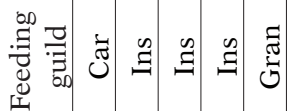

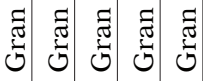

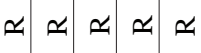

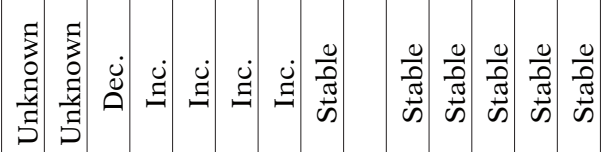

$\begin{array}{llllllll}\cup & \cup & \cup & \cup & \cup & \cup & \cup & \cup \\ \bullet & \ddots & \ddots & \ddots & \ddots & \ddots & \ddots\end{array}$

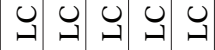

\begin{tabular}{ll|l|l|l|l|l|}
\hline & & & & & & \\
\hline
\end{tabular}

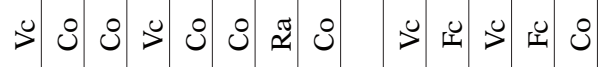

\section{\begin{tabular}{|l|l|l|l|l|l|l|}
\hline & & & & & & \\
\hline
\end{tabular}}

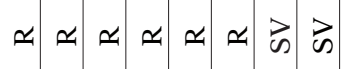

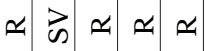

\section{\begin{tabular}{|l|l|l|l|l|}
\hline & & & & \\
\hline
\end{tabular}}

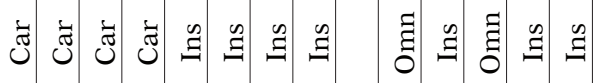

\section{జ్ల్ర}

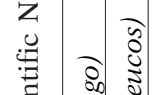

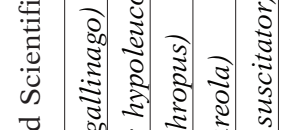

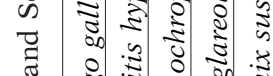

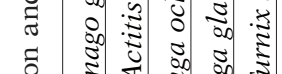

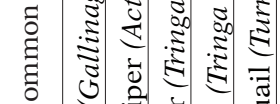

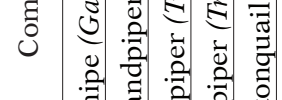

क

จี

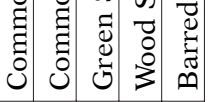

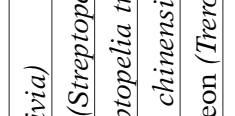

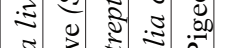

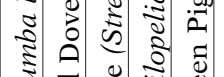

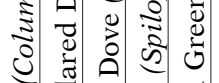

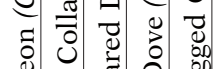

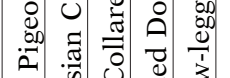

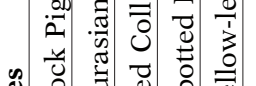

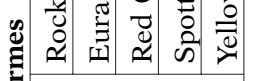

\section{\begin{tabular}{|l|l|l|l|l|l|l|}
\hline & & & & & & \\
\hline
\end{tabular}}

ลิ

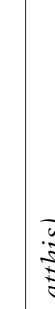

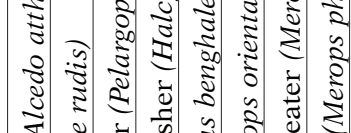

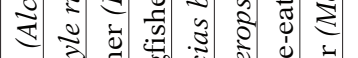

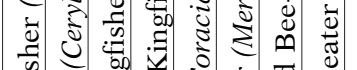

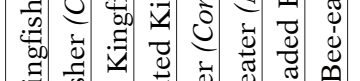

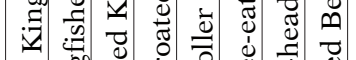

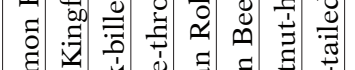

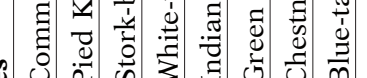

-

जี

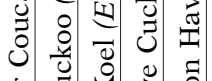

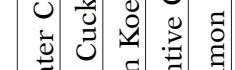

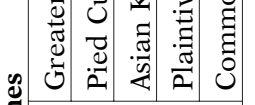




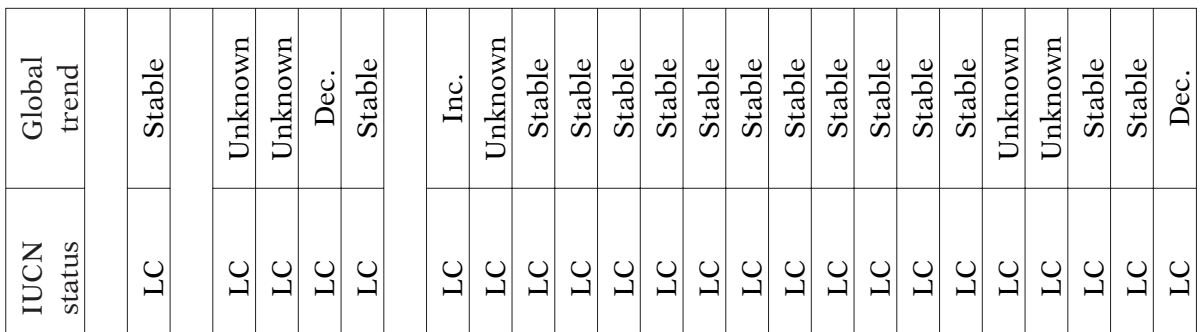

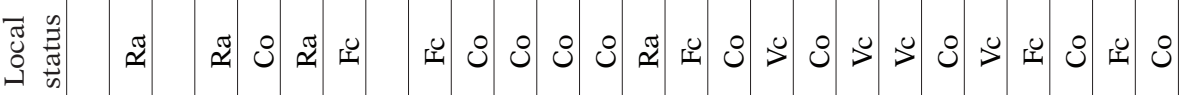

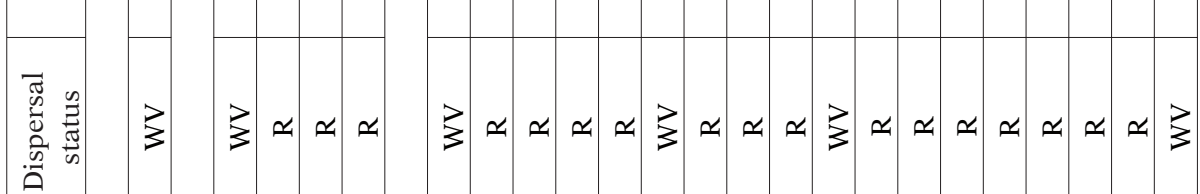

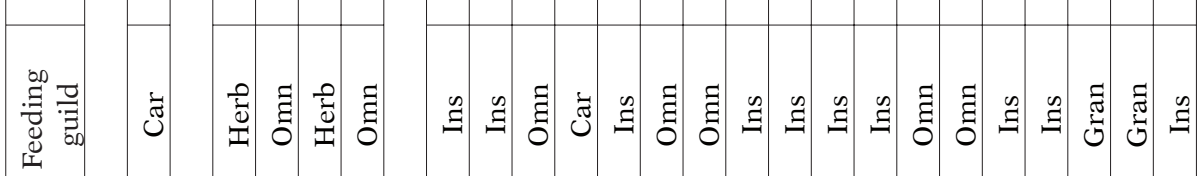

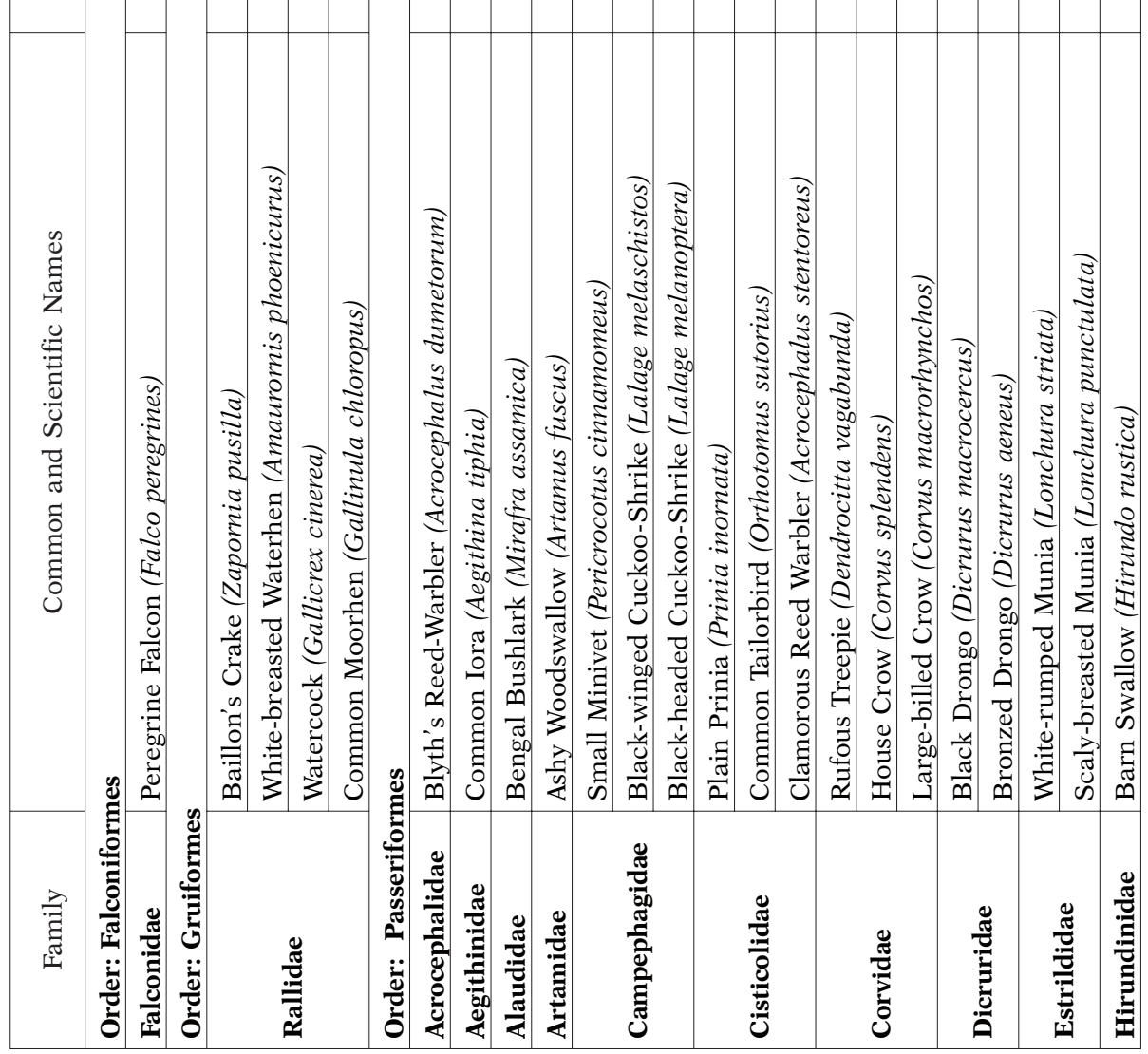




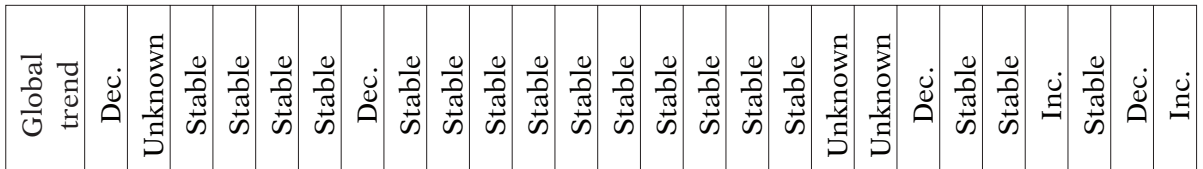

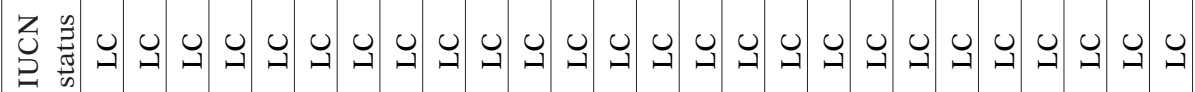

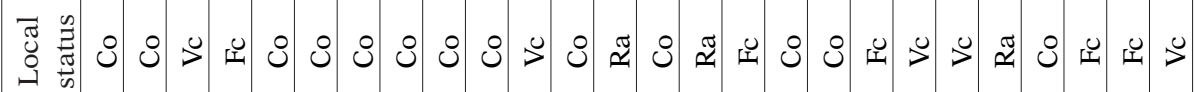

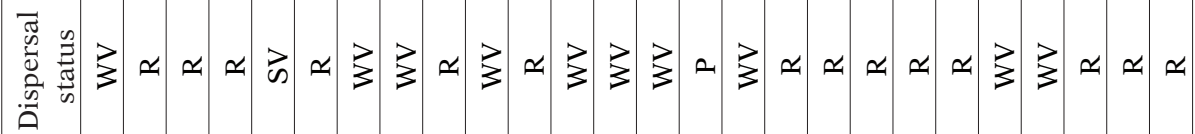

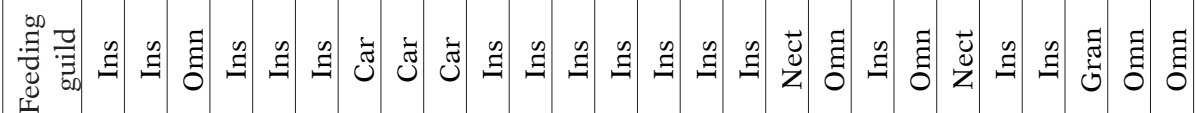

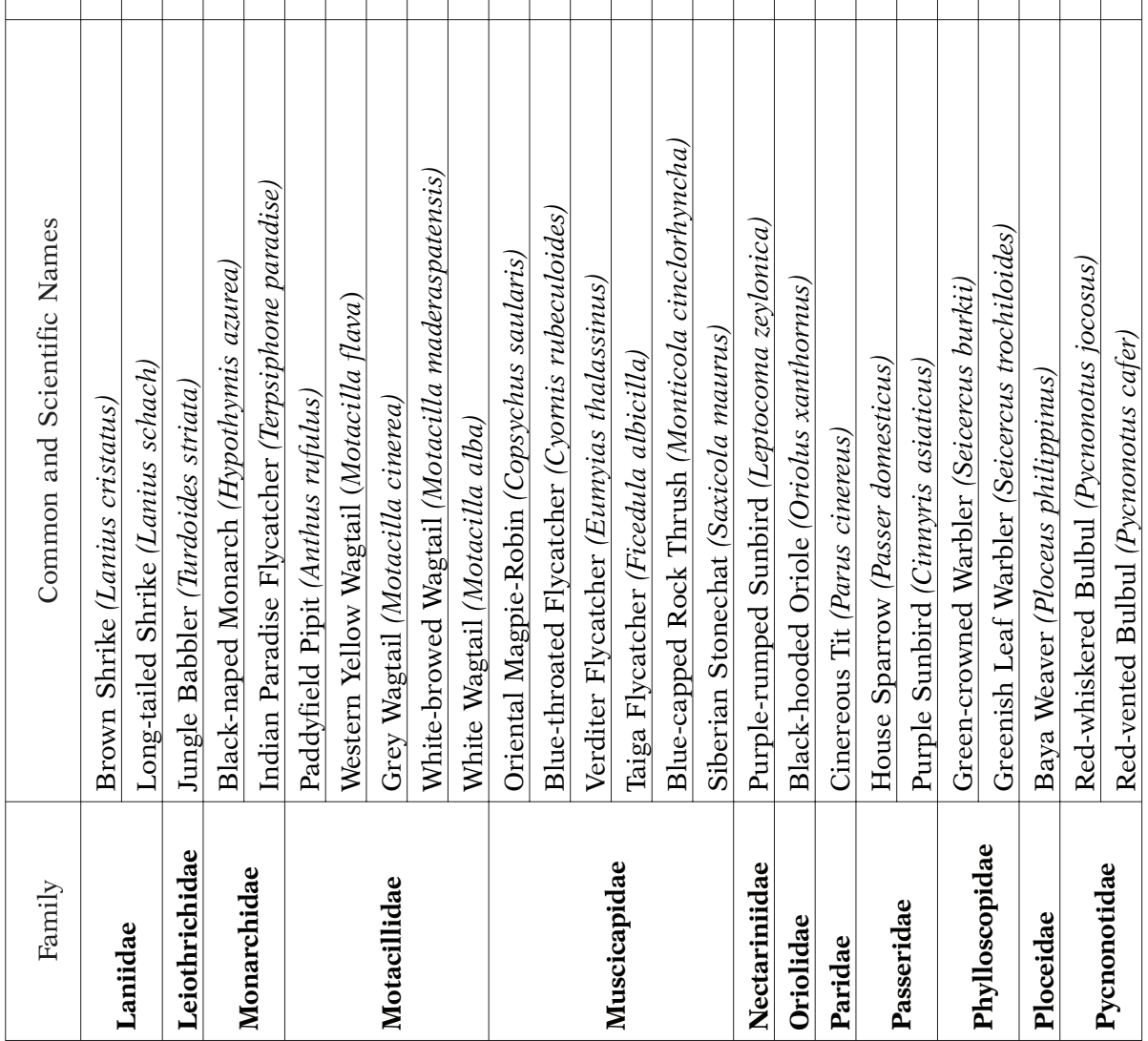




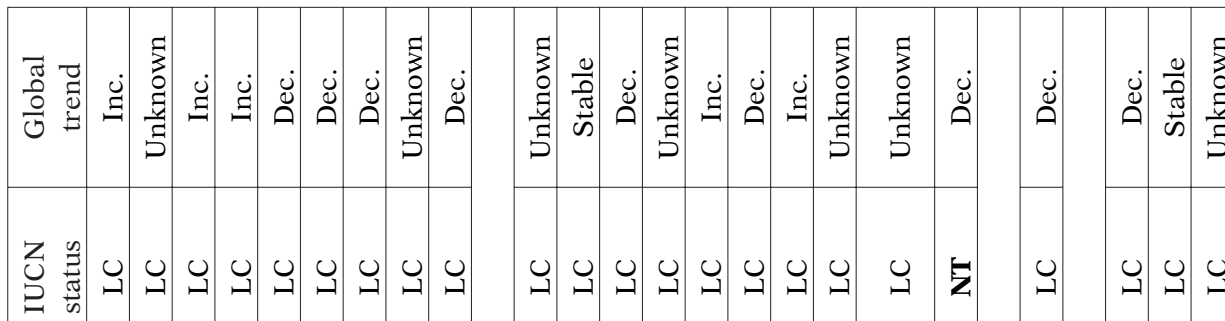

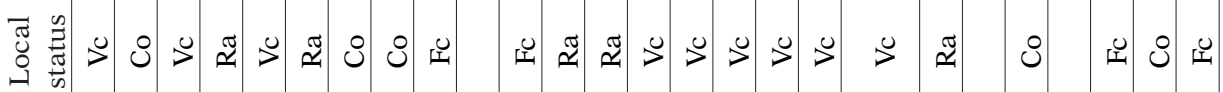

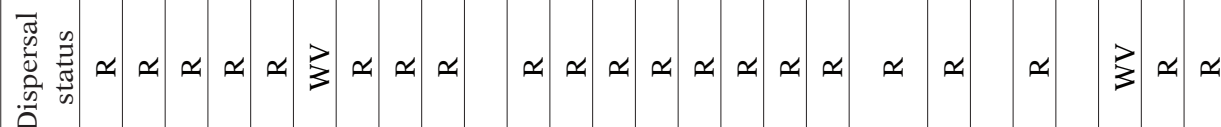

宽苛

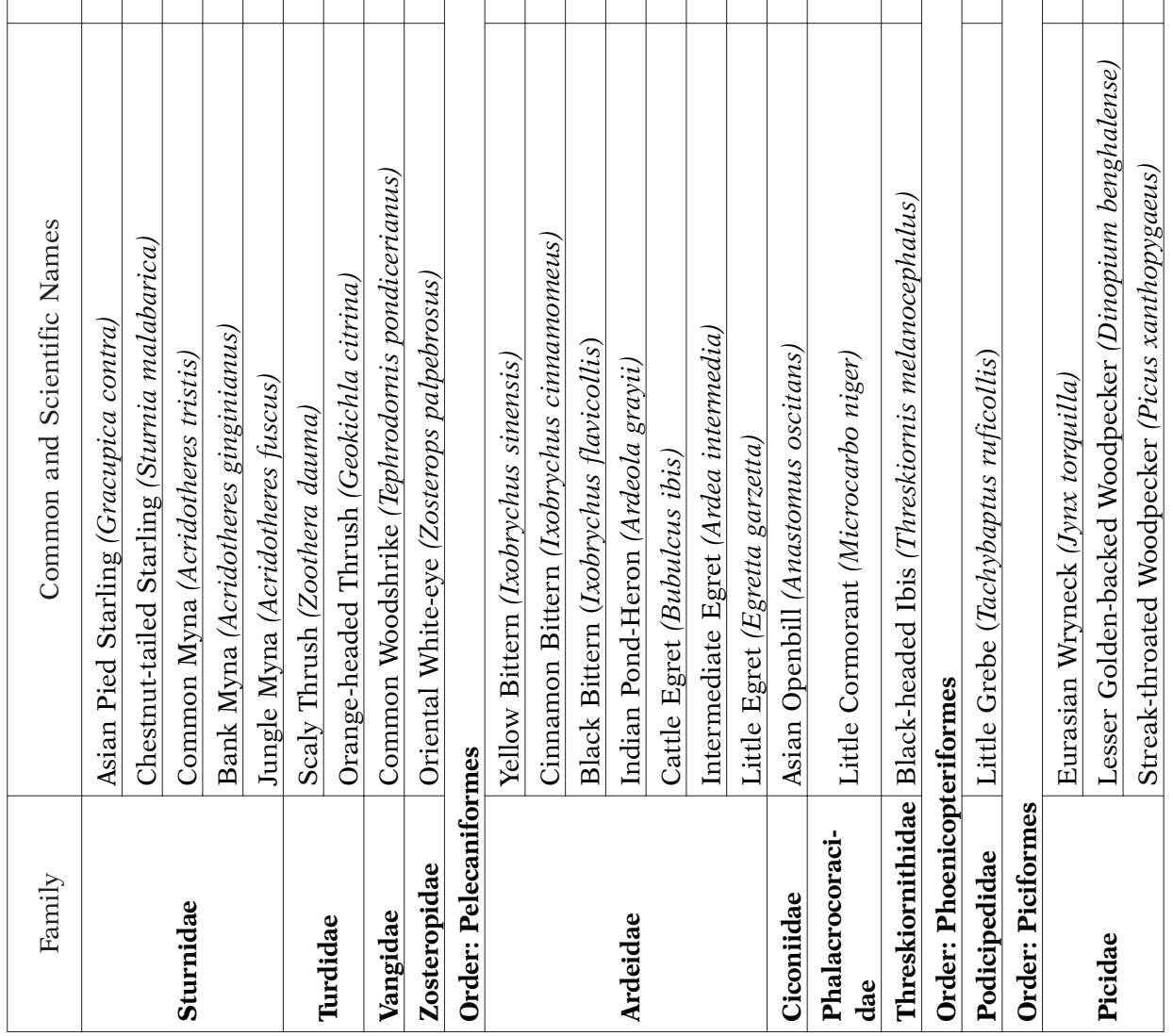




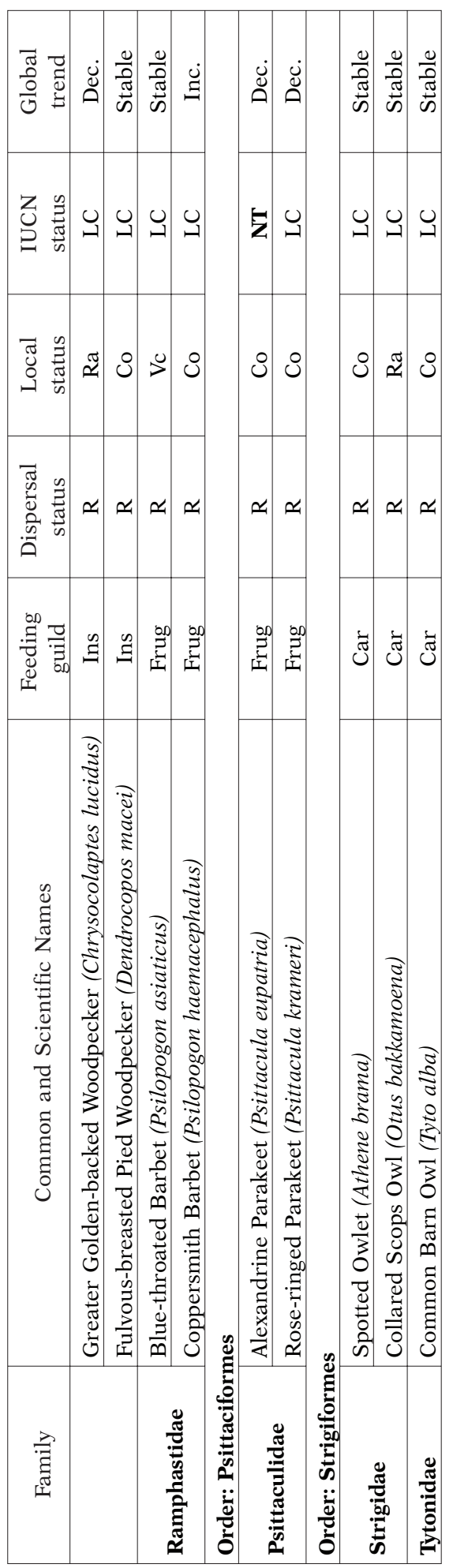


by Muscicapidae (6 species, $R D i=5.04$ ), while 23 families were poorly represented in the study area, with single species from each $(R D i=0.84)$ (Table 2$)$.

Table 2

Relative diversity (RDi) of various avian families in and around Bongaon, West Bengal, India

\begin{tabular}{|l|c|c|}
\hline \multicolumn{1}{|c|}{ Avian Families } & Number of species & RDi value \\
\hline Ardeidae & 7 & 5.882 \\
\hline Muscicapidae & 6 & 5.042 \\
\hline Columbidae, Cuculidae, Motacillidae, Picidae, Sturnidae & 5 & 4.202 \\
\hline Alcedinidae, Rallidae, Scolopacidae & 4 & 3.361 \\
\hline $\begin{array}{l}\text { Anatidae, Campephagidae, Charadridae, Cisticolidae, } \\
\text { Corvidae, Meropidae }\end{array}$ & 3 & 2.521 \\
\hline $\begin{array}{l}\text { Apodidae, Dicruridae, Estrildidae, Jacanidae, Laniidae, } \\
\text { Monarchidae, Passeridae, Phylloscopidae, Psittaculidae, } \\
\text { Pycnonotidae, Ramphastidae, Strigidae, Turdidae }\end{array}$ & 2 & 1.681 \\
\hline $\begin{array}{l}\text { Accipitridae, Acrocephalidae, Aegithinidae, Alaudidae, } \\
\text { Artimidae, Caprimulgidae, Ciconiidae, Coraciidae, Falco- } \\
\text { nidae, Hirundinidae, Leiotrichidae, Nectariniidae, Orioli- } \\
\text { dae, Pandionidae, Paridae, Phalacrocoracidae, Ploceidae, } \\
\text { Podicipedidae, Turnicidae, Threskiornithidae, Tytonidae, } \\
\text { Upupidae, Vangidae, Zosteropidae }\end{array}$ & 1 & \\
\hline
\end{tabular}

Species richness was highest in November $(68.7 \pm 3.01)$ and lowest in July (49.8 \pm 0.63$)$. In the study area, 89 species (74.8\%) were resident, 25 species $(21.0 \%)$ were winter visitors, four species $(3.4 \%)$ were summer visitors and one species $(0.8 \%)$ was a passage migrant. Species richness of resident avifauna showed no significant variation between seasons (Kruskal-Wallis test: $K=5.024$, $d f=3, p=0.17$ ), remaining similar throughout the study period (Fig. 2). Blue-headed Rock-Thrush was the passage migrant, which also did not vary seasonally (Kruskal-Wallis test: $K=3, d f=3, p$ $=0.39$ ) and was noted only once during the study period. On the other hand, significant seasonal difference was noticed in the case of winter visitors (Kruskal-Wallis test: $K=60.708, d f=3, p<0.05$ ) and summer visitors (Kruskal-Wallis test: $K=$ $41.408, d f=3, p<0.05)$. The number of winter visitors significantly increased in the post-monsoon season, reached its peak in winter and then sharply declined in summer (Fig. 2). Multiple pair-wise post-hoc comparisons (two-tailed tests) using Dunn's procedure revealed that the species richness of winter visitors in summer and the monsoon season was significantly lower $(p<0.05)$ than in the post-monsoon season and winter. In contrast, the species richness of summer visitors was highest in summer and differed significantly $(p<0.05)$ from all other seasons (Fig. 2).

Analysis of the feeding guilds of the 119 species revealed that 49 species $(41.2 \%)$ were insectivorous, $29(24.4 \%)$ were carnivorous, 22 (18.5\%) were omnivorous, $9(7.6 \%)$ were granivorous, 4 species $(3.4 \%)$ each were frugivorous and herbivorous, and $2(1.7 \%)$ were nectarivorous. The species richness of these feeding guilds varied significantly (Kruskal-Wallis test: $K=16.812, d f=6, p<0.05$ ). Multiple pair-wise post-hoc comparisons using Dunn's procedure (two-tailed tests) also revealed signifi- 
cant differences between each pair of feeding guilds in the study area (Bonferroni corrected significance: $p<0.05$ ), except between insectivores and omnivores (Bonferroni corrected significance: $p>0.05$ ), as shown in Figure 3.
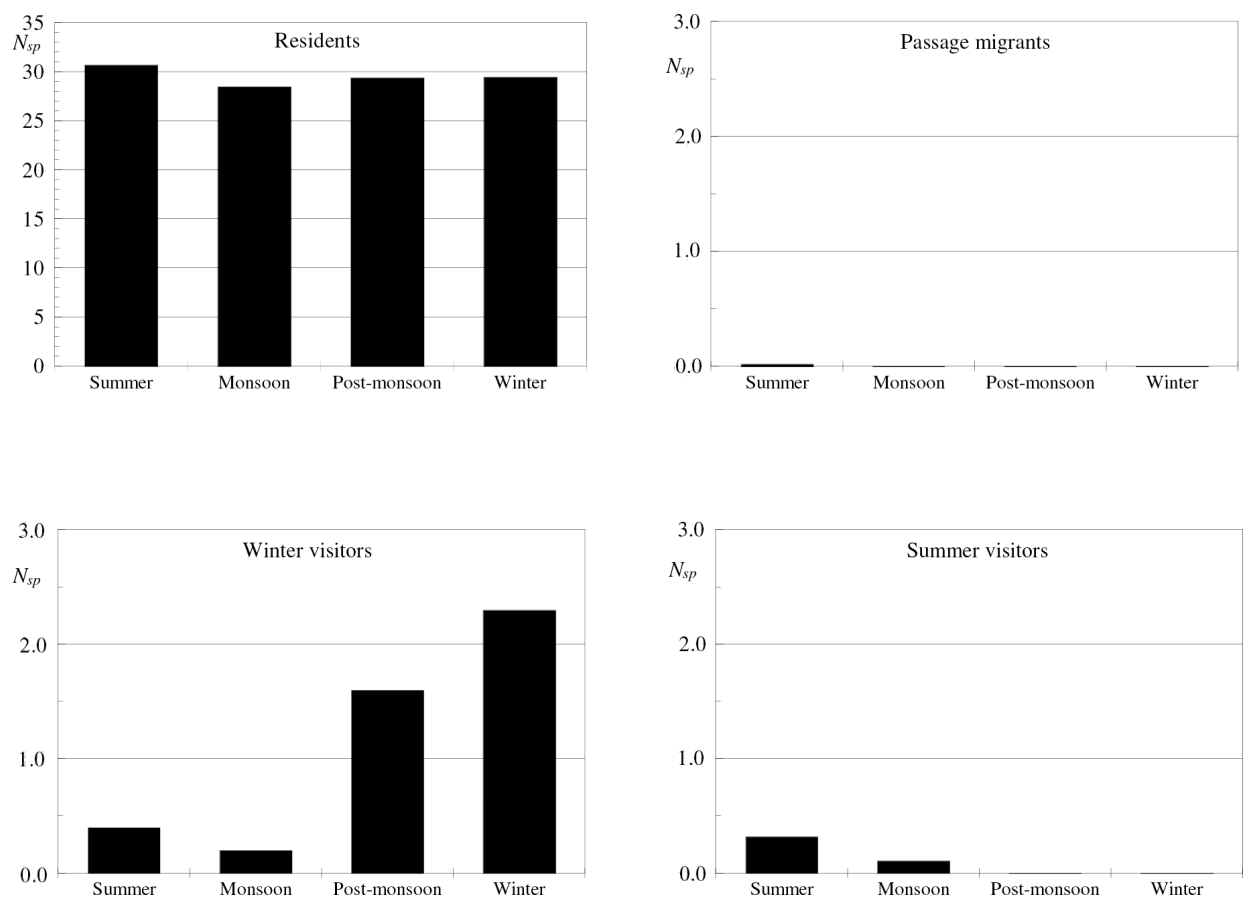

Fig. 2. Changes in numbers of bird species (resident, passage migrant, winter visitor and summer visitor) in suburban area of Bongaon, West Bengal, India

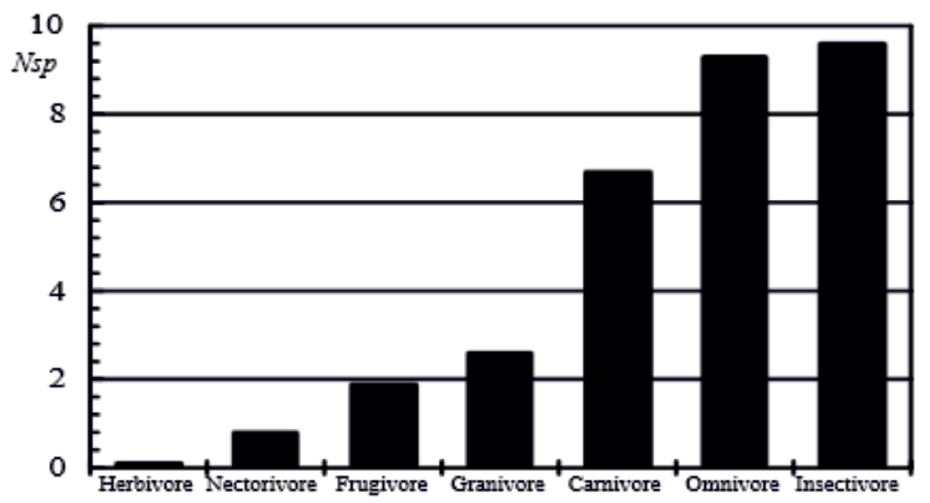

Fig. 3. Numbers of bird species belonging seven foraging guilds (i.e. herbivore, nectarivore, grugivore, granivore, carnivore, omnivore and insectivore) recorded from the suburban area of West Bengal, India 
Among all avian species observed during the study, only two (Black-headed Ibis and Alexandrine Parakeet) fall under the IUCN near-threatened (NT) category, while the remaining species are categorized as least concern (LC) species (del Hoyo et al. 2014). Assessment of local abundance revealed that 22 species (18.5\%) were very common, 52 species $(43.7 \%)$ were common, 26 species $(21.8 \%)$ were fairly common and 19 species $(16.0 \%)$ were rare. When this local abundance was compared with the global population trend for the species (del Hoyo et al. 2014), we found that six species having a globally declining trend were still very common in the study area (Fig. 4).

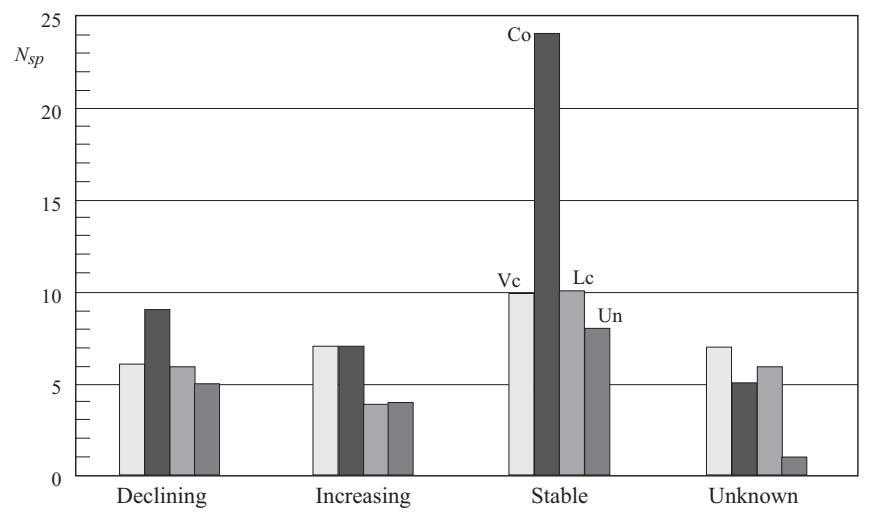

Legends: Vc - very common, Co - common, Lc - less common, Un - uncommon) vs. global population trends (> - decreasing, <- increasing, = - stable; ? - unknown

Fig. 4. Comparison of the local abundance (Vc, Co, Lc and Un) and global population trend (dec, inc, stable and unknown) of the avian species recorded from the suburban area of Bongaon, West Bengal, India

\section{DISCUSSION}

Urban sites have a greater proportion of avian species which are multiple brooders, construct nests on buildings and other urban structures, eat seeds, reside year round and are non-territorial. In contrast, natural sites have a greater proportion of individuals that are single brooders, nest in shrubs and snags, eat insects, migrate long distances and maintain territories during the breeding season. Suburban habitats are tipping points in the shift of avian communities from wilderness areas to exotic and homogeneous urban landscapes (Blair and Johnson 2008). The suburban area we surveyed supports $12.7 \%$ of the avian species found in West Bengal $(N=937)$, and this richness of avifauna is comparable with many ecosystems of this state. For instance, Roy et al. (2011) studied avifaunal diversity in three different national parks and a forest reserve in North Bengal and recorded 117 bird species belonging to 42 families; Dubey et al. (2015) noted 99 species belonging to 43 families from Jaldapara National Park; and Patra and Chakrabarti (2014) reported 86 species belonging to 10 orders and 35 families around Digha in West Bengal. Perhaps the heterogeneity of habitats in the study area augments the availability of diverse resources. Some other 
studies have also reported higher biodiversity in regions with human interference (Pautasso 2007).

The greatest number of avian species in the study area belonged to the order Passeriformes. According to Praveen et al. (2016), passerines (order Passeriformes) form the most predominant avian taxon in India (about 54\%). Muscicapidae is the largest family of birds in India (Manakadan and Pittie 2001). In our study area, however, the Ardeidae showed the highest diversity of species, followed by the Muscicapidae. Nevertheless, several studies have also found Ardeidae to be the most diverse avian family, particularly in wetland habitats (Vijayan et al. 2006, Kumar 2006, Surana 2007, Zakaria et al. 2009, Zakaria and Rajpar 2010, Ali et al. 2011, Zhang et al. 2012, Dal and Vaghela 2015, Mazumdar 2017).

The high species richness of the assemblage in the study area may be due to the presence of diverse habitats in and around this suburban area. The landscape of the area comprises of built-up areas together with wetlands, riverbanks, vast stretches of paddy cultivation, and monoculture plantations of Shorea and Eucalyptus, as well as uncultivated grazing pastures, backyard gardens, orchards and bamboo thickets. Such habitat diversity in this suburban area plays a crucial role in supporting fairly high species richness. Resident species were present throughout the year and showed no seasonal variation, but the migratory species (winter visitors and summer visitors) showed a definite species-specific pattern of arrival and departure from the study area. The species richness of this suburban area begins to increase with the arrival of winter migrants. Winter visitors began appearing in September, increasing the avian diversity in winter.

The fundamental requirements of migratory birds in the wintering site include climate and a favourable habitat ensuring food availability and safety. The perennial wetland attracts and serves as a wintering grounds for migratory birds (especially migratory ducks) every year, and also supports large breeding populations of the Pheasant-tailed Jacana and the Bronzed-winged Jacana, which were seen to thrive on floating vegetation (dominated by water hyacinth). The floating macrophytes and fauna (e.g. fish, crabs, snails and clams) of this wetland serve as food for many species of avifauna. The species richness of the area increases again in the summer months (highest in May) due to the appearance of four species of summer visitors. A monoculture plantation forest in the study area serves as a safe breeding site for some resident (Changeable Hawk Eagle, Small Minivet, Common Woodshrike, Common Iora, Common Myna, Jungle Myna, Chestnut-headed Starling, Asian Pied Starling, Spotted Dove, and Red-vented Bulbul) and migrant (Asian Paradise Flycatcher and Chestnut-headed Bee-eater) birds.

The rich avian assemblage of this suburban area (Bongaon, West Bengal, India) also reflects possible variation in their functional roles, feeding habits and resource utilization pattern. The greatest number of species within the area were insectivores, indicating rich abundance of insects here. These insectivorous birds play a crucial role in the biocontrol of various insect pests thriving in agriculture, horticulture and forests (Mahabal 2005, Thakur et al. 2010) in the adjoining areas. Indiscriminate use of chemical pesticides in the adjoining paddy fields could have severe ecological consequences and a grave effect on the avifauna of this suburban area. Insectivorous 
birds often consume insects contaminated with pesticides (Sánchez-Bayo et al. 1999), and thus these birds, being at a higher trophic level, are at high risk of suffering from toxic effects of bioaccumulation of such chemicals (Sánchez-Bayo 2011). On the other hand, if a pesticide eliminates enough of their prey base, then insectivorous birds could die of malnutrition or starvation rather than poisoning (Campbell and Cooke 1997, Tiebout III and Brugger 1995). Hence, monitoring of the insectivorous species of this suburban area may reflect the impact of pesticide use on this avian guild.

It is evident that birds in various habitats are under threat due to increased anthropogenic activity resulting in habitat destruction and fragmentation (Baral and Inskipp 2005, Gautam and Kafle 2007). Accelerated urban development in this suburban area, involving filling in ponds and water bodies and cutting down trees to widen roads and for other developmental purposes, leads to habitat alteration (pers. obs.), thereby reducing the safe refuges of many common birds. Local people also informed us that some avian species, such as egrets, openbill storks, sandpipers, snipes, or White-breasted Waterhen, are occasionally hunted for food. Ecologically, birds are of tremendous importance to human society. Birds are a good medium for dispersing seeds, pollinating plants, biological control of pests, and thus have an important role in continuing the ecological cycle. Hence a decline in the diversity of birds may induce a cascading effect on the food chain, affecting multiple species and subsequently disrupting the species interactions and integrity of ecosystem functions (Sekercioglu et al. 2004). Regular monitoring of avifauna is, therefore, an excellent means of keeping watch on ecosystem health. Interestingly, six species with a globally declining population trend (Lesser Whistling-Duck, Intermediate Egret, Rock Pigeon, House Sparrow, Jungle Myna and Alexandrine Parakeet) were found to be very common in our study area, which indicates that favourable resources for these birds are still available in this suburban area. These species must be prioritized for regular and long-term monitoring from a global bird conservation perspective.

In India, documentation of the species-specific roles and ecological services of diverse avifauna in various ecosystems is close to the nascent stage (Dhindsa and Saini 1994, Singh and Bayal 2013, Gopisundar and Kittur 2013, Sengupta et al. 2014). Documentation of the species richness and composition of birds in a particular landscape is a prerequisite to assess of their ecological importance. In this context, the present study is the first scientific documentation of the assemblage and richness of avian species in a suburban area of West Bengal, India. Monitoring of the avian community should be continued in the study area, as it may provide an early indication of whether this ecosystem suffers from any detrimental change. Our study showed that this suburban area is a promising region for ecological and behavioural research on avifauna. Our findings on the avian composition of this area can be used for further ecological assessment of the avian community. Nevertheless, detailed studies should be undertaken, focusing on the population abundance, habitat use, nesting, breeding and foraging ecology of the birds, in order to understand their critical role in performing various ecosystem services. Assessment of species-specific roles for monitoring environmental changes, as well as documentation of threats to diverse avifauna, may also be useful in bridging the gap of existing knowledge on avifauna and sustaining the ecosystem integrity of this suburban landscape. 


\section{ACKNOWLEDGMENTS}

We are indebted to Dr. Sanjay Poddar of Barasat Government College for his constant encouragement. Permission and infrastructural facilities provided by Principal, Barasat Government College and Principal, Shibpur Dinabundhoo Institution (College) are gratefully acknowledged. Special thanks are due to Mr. Shaktidev Mishra, Mr. Sourav Mondal and Mr. Sourabh Biswas for their help during the survey.

Funding Information: We bore all expenses during the research and did not receive any grant from funding agencies.

\section{REFERENCES}

Ali S., Ripley S.D. 1987. Compact handbook of Birds of India and Pakistan together with those of Bangladesh, Nepal, Bhutan, and Srilanka. Oxford University Press.

Ali Z., Bibi F., Shelly S.Y., Qazi A., Khan A.M. 2011. Comparative Avian Faunal Diversity of Jiwani Coastal Wetlands and Taunsa Barrage Wildlife Sanctuary, Pakistan. J. Anim. Plant Sci. 21(2 Suppl.): 381-387.

Baral H.S., Inskipp C. 2005. Important bird areas in Nepal: key sites for conservation. Bird Conservation Nepal and Birdlife International.

Bensizerara D., Chenchouni H., Bachir A.S., Houhamdi M. 2013. Ecological status interactions for assessing bird diversity in relation to a heterogeneous landscape structure. Avian Biology Research 6(1): 67-77.

Bibby C.J., Burgess N.D., Hill D.A., Mustoe S.H. 2000. Bird census techniques. Academic Press.

BirdLife International. 2010. IUCN Red List for birds. Available from: http://www.birdlife.org

Blair R.B., Johnson E.M. 2008. Suburban habitats and their role for birds in the urban-rural habitat network: points of local invasion and extinction? Landsc. Ecol. 23: 1157-1169.

Campbell L., Cooke A. 1997. The Indirect Effects of Pesticides on Birds. Joint Nature Conservation Committee.

Chen I.C., Hill J.K., Ohlemuller R., Roy D.B., Thomas C.D. 2011. Rapid range shifts of species associated with high levels of climate warming. Science 333: 1024-1026.

Crooks K.R., Suarez A.V., Bolger D.T. 2004. Avian assemblages along a gradient of urbanization in a highly fragmented landscape. Biol Conserv. 115: 451-462

Dal P., Vaghela A.K. 2015. Preliminary survey of avifaunal diversity around Shetrunji River, Dhari, India. J. Biol. Earth Sci. 5(1): 19-24.

Dhindsa M.S., Saini H.K. 1994. Agricultural ornithology: An Indian perspective. J. Biosci. 19(4): 391-402.

Dubey S.K., Chakraborty D.C., Gupta S., Mitra M., Bhattacharya R., Neogy A.B. 2015..Avian Diversity in the Jaldapara National Park, West Bengal, India with a Notes on their Habitat Association and Feeding Guild. Indian Forester 141(10): 1092-1101.

Gautam R., Kafle G. 2007. A Preliminary Survey of Waterbirds in Phewa Lake, Kaski. Danphe 16(3/4): 6-8.

Gopisundar K.G., Kittur S. 2013. Can wetlands maintained for human use also help conserve biodiversity? Landscape-scale patterns of bird use of wetlands in an agricultural landscape in north India. Biol. Conserv. 168: 49-56.

Gregory R.D., Noble D., Field R., Marchant J.H., Raven M., Gibbons D.W. 2003. Using birds as indicators of biodiversity. Ornis Hungaria 12-13: 11-24. 
Gregory R.D., Vorisek P., Noble D.G., Strien A.V., Klvanova A., Eaton M., Meyling A.W.G., Joys A., Foppen R.P.B., Burfield I.J. 2008. The generation and use of bird population indicators in Europe. Bird Conserv. Int. 18: S223-S244

Grimmett, R., Inskipp C., Inskipp T. 2011. Birds of the Indian Subcontinent ( $2^{\text {nd }}$ edn.), Oxford University Press \& Christopher Helm.

Hansen A.J., Knight R.L., Marzluff J.M., Powell S., Brown K., Gude P.H., Jones K.B. 2005. Effects of exurban development on biodiversity: patterns, mechanisms, and research needs. Ecol. Appl. 15: 1893-1905.

Hutto, R.L. 1985. Habitat Selection in Birds. Academic Press. Inc.

Khan M.S., Syed A.Z., Nawab O., Ilyas O., Khan A. 2013. Composition and conservation status of avian species at Hastinapur Wildlife Sanctuary, Uttar Pradesh, India. JoTT.. 5: 4714-4721

Khan S.I., Naher H. 2009. Birds in Kurigram district of Bangladesh. JoTT. 1(4): 245-250.

Kumar A.B. 2006. A checklist of avifauna of the Bharathpuzha river basin, Kerala. Zoos Print 21(8):2300-2355.

Lawson J.H., Bignell D.E., Bolton B., Bloemers G.F., Eggleton P., Hammond P.M., Hodda M., Holt R.D., Larsen T.B., Mawdsley N.A., Stork N.E., Srivastava D.S., Watt A.D. 1998. Biodiversity inventories, indicator taxa, and effects of habitat modification in tropical forest. Nature 391:72-76.

Mahabal A. 2005. Aves. In: The Director (ed.). Fauna of Western Himalaya, Zoological Survey of India, Kolkata, pp. 275-339.

Manakadan R., Pittie A. 2001. Standardised common and scienti?c names of the birds of the Indian Subcontinent. Buceros 6:1-37.

Marzluff J.M., Bowman R., Donnelly R. (eds.). 2001. Avian Ecology and Conservation in an Urbanizing World. Kluwer Academic Press, Norwell.

Mazumdar S. 2017. Compositions of avian communities in a human-modified wetland Okhla Bird Sanctuary, India: with notes on conservation initiatives. Proocedings of the Zoological Society. https://doi.org/10.1007/s12595-017-0239-6.

McKinney M.L., Lockwood J.L. 2001. Biotic homogenization: a sequential and selective process. In: Lockwood J.L., Mckinney M. (eds.). Biotic Homogenization, Kluwer Academic, New York, pp. 1-17.

McKinney M.L. 2002. Urbanization, biodiversity, and conservation. Bioscience 52: 883-890.

Miller J.R., Hobbs R.J. 2002. Conservation where people live and work. Conserv. Biol. 16: 330-337

Patra G., Chakrabarti S. 2014. Avian Diversity in and around Digha, District-East Midnapore (West Bengal, India). Advances in Bioscience and Biotechnology 5: 596- 602.

Pautasso M. 2007. Scale dependence of the correlation between human population presence and vertebrate and plant species richness. Ecol. Lett. 10: 16-24.

Pickett S.T.A., Cadenasso M.L., Grove J.M., Nilon C.H., Pouyat R.V., Zipperer W.C., Costanza R. 2001. Urban ecological systems: linking terrestrial ecological, physical, and socioeconomic components of metropolitan areas. Annu. Rev. Ecol. Evol. Syst. 32: 127-157.

Praveen J., Jayapal R., Pittie A. 2016. Checklist of the birds of India. Indian Birds 11(5\&6): 113-172.

Rapoport E.H. 1993. The process of plant colonization in small settlements and large cities. In: McDonnell M.J., Pickett S.T.A. (eds.) Human as component of ecosystems, Springer, New York, pp. 190-207.

Roy U.S., Banerjee P., Mukhopadhyay S.K. 2012. Study on avifaunal diversity from three different regions of North Bengal, India. Asian Journal of Conservation Biology 1(2):120-129.

Sánchez-Bayo F. 2011. Impacts of agricultural pesticides on terrestrial ecosystems. In: Sánchez-Bayo F., van den Brink P.J., Mann R. M. (eds.) Ecological Impacts of Toxic Chemicals, Bentham Science Publishers, pp. 63-87.

Sánchez-Bayo F., Ward R., Beasley H. 1999. A new technique to measure bird's dietary exposure to pesticides. Anal Chim Acta., 399:173-183. 
Sekercioglu C.H. 2006. Increasing awareness of avian ecological function. Trends Ecol. Evol. 21: 464-471.

Sekercioglu C.H. 2012. Bird functional diversity and ecosystem services in tropical forests, agroforests and agricultural areas. J. Ornithol. 153(Suppl 1): S153-S161.

Sekercioglu C.H., Daily G.C., Ehlrich P.R. 2004. Ecosystem consequences of bird declines. Proc. Natl. Acad. Sci. U.S.A. 101: 18042-18047.

Sekercioglu C.H., Primack R.B., Wormworth J. 2012. The effects of climate change on tropical birds. Biol. Conserv. 148: 1-18.

Sengupta S., Mondal M., Basu P. 2014. Bird species assemblages across a rural urban gradient around Kolkata. Urban Ecosyst. 17(2): 585-596.

Singh V., Banyal H.S. 2013. Avian fauna of Khajjar Lake, district Chamba, Himachal Pradesh, India. Proc. Zool. Soc. 66(2): 130-136.

Surana R. 2007. Avian diversity during rehabitation stage of chimdi lake, Sunsari Nepal. Our Nature, 5(1): $75-80$.

Sutherland W.J. 2006. Ecological Census Techniques a handbook. Cambridge University Press, New York.

Thakur M.L., Mattu V.K., Lal H., Sharma V.N., Raj H., Thakur V. 2010. Avifauna of Arki Hills, Solan (Himachal Pradesh), India. Indian Birds 5: 162-166

Tiebout III H. M., Brugger K. E. 1995. Ecological Risk Assessment of Pesticides for Terrestrial Vertebrates: Evaluation and Application of the U.S. Environmental Protection Agency's Quotient Model. Conserv. Biol. 9(6): 1605-1618

Torre-Cuadros M.D.L.A.L., Herrando-Perez S., Young K.R. 2007. Diversity and structure patterns for tropical montane and premontane forests of central Peru, with an assessment of the use of higher-taxon surrogacy. Biodivers. Conserv. 16: 2965-2988.

Veech J.A., Small M.F., Baccus J.T. 2011. The effect of habitat on the range expansion of a native and an introduced bird species. J. Biogeogr. 38: 69-77.

Vijayan L., Prasad S.N., Sridharan N., Guptha M.B. 2006. Status of Wetlands and Wetland Birds in Selected Districts of Tamilnadu. Sálim Ali Centre For Ornithology \& Natural History, Coimbatore. http://www.sacon.in/wp-content/uploads/2015/06/FT-2006-PR135s-STATUS-OF-WL-TN.pdf

Western D., Grimsdell J.J.R. 1979. Measuring the distribution of animals in relation to environment - Handbook No. 2 ( $2^{\text {nd }} e d n$.). African Wildlife Foundation, Nairobi, Kenya.

Whelan, C.J., Wenny D.G., Marquis R.J. 2008. Ecosystem services provided by birds. Ann. N. Y. Acad. Sci. 1134: 25-60.

Yamian Z., Yifei J., Shengwu J., Qing Z., Duoduo F., Yumin G., Guangchun L. 2012. Wuliangsuhai wetlands: a critical habitat for migratory water birds. J. Resour. Ecol. 3(4): 316-323.

Zakaria M., Rajpar M.N. 2010. Bird Species Composition and Feeding Guilds Based on Point Count and Mist Netting Methods at The Paya Indah Wetland Reserve, Peninsular Malaysia. Trop. Life Sci. Res. 21(2): 7-26.

Zakaria M., Rajpar M.N., Sajap A.S. 2009. Species Diversity and Feeding Guilds of Birds in Paya Indah Wetland Reserve, Peninsular Malaysia. Int. J. Zool. Res. 5(3): 86-100. 